\title{
Lifetime of Sensor Network by Exploiting Heterogeneity- A Survey
}

\author{
Ritesh Kumar Singh \\ Deptt. of Wireless Communication and Computing, Indian Institute Of Information Technology, Allahabad, India \\ Email: ritesh.iiit10@gmail.com
}

Ankit Bhadoria

Deptt. of Computer Science and Engineering, National Institute of Technology, Hamirpur, India

Email:ankbhadoria@gmail.com

\begin{abstract}
Wireless sensor networks (WSN) are emerging in various fields. A large number of sensors in these applications are unattended and work autonomously. Lifetime is an important parameter which is critical for different algorithms for data transfer. Moreover it is responsible for the throughput and the failure of the network. Heterogeneous wireless sensor network, on top of clustering technique, has evolved as the major parameter to increase the lifetime of the Sensor network, data transfer, energy consumption and the scalability of the sensor network. This paper surveys the different clustering algorithm and dependencies for heterogeneous wireless sensor network. This paper is for scholars to gain sufficient knowledge of wireless sensor network (WSN), its important characteristics, and performance metrics with factors responsible for a WSN system. It can help a scholar to start a quick research by understanding all the respective parameters and energy oriented strategies in WSN.
\end{abstract}

Index Terms - Cluster head, Heterogeneous, Energy Efficient, Wireless Sensor Network.

\section{INTRODUCTION}

It has been noticed the great growing interest towards the Wireless Sensor Networks (WSNs). Wireless Sensor Networks are widely used in huge sensing areas for example real life applications like robotics, aircrafts, disaster management, medical, border security surveillance and many more. As Wireless Sensor Network has unrechargeable and very limited and less energy resources, it gives birth to the research in enhancing or increasing the lifetime of Wireless Sensor Networks, In some of the cases sensor nodes with comparatively different perspective in terms of high energy or functionality can be used (which makes the WSNs heterogeneous) in order to prolong the lifetime of Wireless sensor Networks. Above all now days every application comprises of mixed nodes, so we need to work to increase the lifetime of system using Heterogeneous Wireless Sensor nodes. Around most of energy efficient protocol, that are designed for the heterogeneous sensor networks are basically based on the clustering techniques, which are effective in saving energy for Wireless sensor networks, In a WSN, sensor nodes are mostly operated through batteries, which are of course limited in energy potential and difficult or even sometimes much impossible to make them replace or recharged. For this particular reason, power should be controlled in a most efficient manner to make maximum use of the limited energy resources, so as to minimize the energy consumed by sensor nodes for some application and thus prolong network lifetime. Especially for this particular purpose, energy efficiency of the sensor nodes must be taken in account in every aspect of operation and network design, not only for few individual sensor nodes, but also considering the communication of the entire network [1]. Power control and Energy efficiency are the basic guarantee of any network performance for example, throughput and delay.

The paper is organized as follows. In section II, the overview of wireless sensor network (WSN) is discussed. In section III, detailed description of various WSN dependent metrics is presented. In section IV, all the factors are described that affects WSN. In section V, all the different definitions of calculating the lifetime of WSN is given. In section VI impact of heterogeneity over WSN is shown. In section VII, factors by which performance of the WSN can be calculated are discussed. In section VIII, energy oriented strategies are explained. Finally, in section IX conclusions are drawn.

\section{VERVIEW OF WIRELESS SENSOR NETWORK}

The act of sensing events has been encouraged from some of biological living creatures. We can say these Sensors have been in the universe for few of the decades now .The sensors are being used in everyday life [2]. Well know applications of sensors include automobiles, aerospace, robotics, smart spaces machines, medical systems etc. With improvement in nanotechnologies like Micro electromechanical System, sensors are now manufactured at microscopic level and moreover at very small prices. In recent time, micro-sensors have attained drastically superior speeds and superior sensitivity in contrast to macro-sensor. Due to such growth in industrialized technologies, with enhancement and rise in 
wireless communication and computing processes, the sensor research has gone through a revolution.

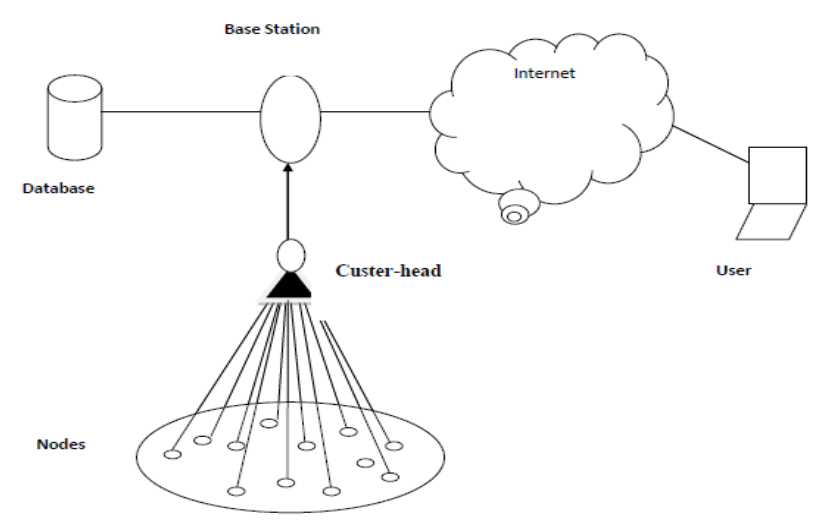

Fig. 1: Wireless Sensor Network Architecture.

Fig. 1 depicts the architecture of the wireless sensor network. The old and conventional system with single sensor is substituted by huge number of small, automatic and self-powered sensors which can be wirelessly linked to the next world via base station [2]. These large numbers of sensors are added into the system mostly for improving performance and lifetime. With so many growing technologies, Civilian, military and many other institutions, are putting in more Labs, scientist, researchers to do more narrow research and develop more efficient sensor network. For a case, we can also use single sensor in order to cover the whole area of our region, but it is much costly and risky as well and it gives sometimes single point of failure, as only one sensor is supposed to cover all area. So providing multiple sensors clearly shorts down many problems in wireless sensor network mainly as lifetime, single point of failures and short communication range etc. Considering for example, cloud of UAVs (Unmanned Aerial Vehicles) gives better condition alertness than of single UAV deployed for reconnaissance or observation. Thus, usually single sensor used for sensing in a system is substituted by many spatially spread sensors, which not only sense interested region but also compute and communicate vital data and resolution to a far station. This is done by combining memory, processors and RF communication devices onto a single board with battery.

\section{SyStem EVAlUATION METRICS}

We have explored that the evaluation metrics that can be used to evaluate the whole wireless sensor network. The Key evaluation metrics, for the whole wireless sensor networks are the coverage, cost, lifetime, response time, temporal accuracy, ease of deployment, security and effective sample rate.

\section{A. Lifetime}

For any of the wireless sensor network, main and important feature is the deployment, which can be affecting, the total expected lifetime. Main limiting factor, which is the issue of lifetime in the sensor network, is due to the limitation in the energy supply. In a sensor node mainly radio consumes majority of the systems energy. The Power consumption is mainly minimized through the decreasing of the transmission power or by working over duty cycle.

\section{B. Coverage}

It is important to keep in mind that the whole coverage of the network, will not always be equal to the total range of the wireless communication links that are being used. The Multi-hop communication techniques can be extended, so as to have the coverage for the network, which is well beyond the range for the radio technology itself and In theory, they have the ability to extend network range in an indefinitely. But, for the approximate or the given transmission ranges the most of the multihop networking protocols increases the overall power consumption for the nodes and which may in turn affect the network lifetime.

\section{Cost and ease of deployment}

The main advantage of the wireless sensor networks is their ease of deployment. The person doing the deployment will not understand the cost, so it should be deployed in a way that the cost of the overall system, should is maintained. For the system deployment and to make it the successful, whole wireless sensor network should have the capability to configure itself.

\section{Response Time}

Now days many of the users have a vision for the wireless sensor networks, as a very useful tool, mainly in the domain of the industrial process control. The, system requires guarantee of response time, an important aspect, that would be required, if it wants to be of any importance. The important feature and the ability to have low response time conflicts can be used, with many existing techniques to increase the network lifetime. The way in which network lifetime can again be increased, is by providing the nodes, techniques that the nodes only operate their radios in certain brief period of time.

\section{E. Temporal Accuracy}

In some of the environmental and many other tracking applications, the samples from multiple nodes, should be such that it can be cross-correlated with time, so as to determine nature of the result that is being measured. The important aspect that is accuracy for this correlation mechanism will be depending mostly on the rate of the propagation for the phenomenon that is being measured.

\section{F. $\quad$ Security}

In spite of the harmless nature of simple application results, like for temperature and light information, from the area like environmental reporting application, keeping this information in a secure manner can be extremely important and useful. If this information goes to the wrong hands then this information can be misused to plan 
a physical attack may be on the company. The Wireless sensor networks should be capable of tracking and keeping the information, so that they are immune from any sort of eavesdropping and if we consider the security oriented applications then the, data security becomes of more significant.

\section{G. Effective Sample Rate}

In the data collection network, the effective sample rate is always a primary application performance metric.

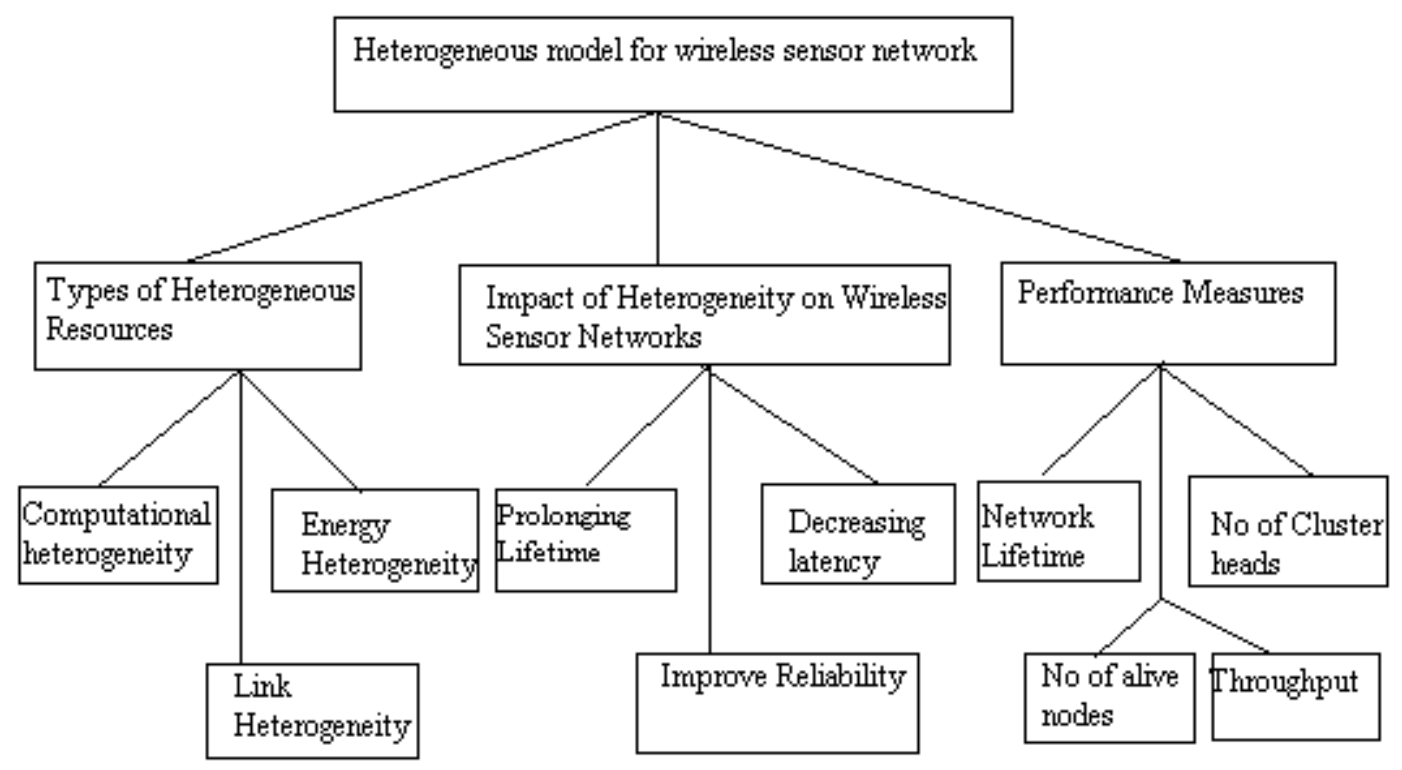

Fig. 2: Heterogeneous Model of WSN

\section{ACtORS AFFECTING WIRELESS SENSOR NETWORK}

In the sensor network, there are many factors that affect the sensor network. These factors play important role in the different overall structures and for different applications. When we design different types of schemes, these factors help us to compare these schemes. Before designing any protocol, there is some study of the dependency parameters which are basically dependent on the factors influencing or responsible for the wireless sensor network. The major impact of the Heterogeneous WSN is reflected by the model shown in Fig. 2.

\section{A. Fault tolerance [3]}

The sensor nodes may die or stop working or can be blocked due to many reasons like lack of power or can face some sort of physical damage or there can be any type of environmental interference. These drawbacks put great impact on the reliability or it is better said to be fault tolerance issue. For the sensor network, the respective Protocols and the algorithms should be designed in such a way so as to address all the possibilities. that can result to fault tolerance. For definition, the Fault tolerance can be said to be an ability to keep on working the functionalities of the respective sensor network, without any sort of the interruption, for any reason like sensor node failures. If we take an example, that if the sensor nodes are being deployed in a room so as to keep track for the parameters like humidity or temperature levels, then the fault tolerance will be very low as in this type of applications the probability of the sensors to die is really low, as they are kept in the room and now if we take the condition that the sensors are deployed in the battle field or any disastrous area then there is high fault tolerance as sensor can die very soon or there can be some sort of malfunctioning that can arise, resulting in the wrong report. It is seen that the fault tolerance mainly depends on the applications so before developing any protocol it should be kept in mind that how is the fault tolerance is going to work in this case for the application.

\section{B. Scalability}

As per the requirements of the application, the total number of sensors may reach the extreme value of thousands. The total number of the sensor nodes that are deployed, for an application may go in the order of hundreds or may be thousands. The protocols designed must utilize the criteria of high density nature of the wireless sensor network. The total density of the network can range from a few sensor nodes to an extreme of few thousands of sensor nodes, in a region and which can be deployed in less than $10 \mathrm{~m}$. For some of the applications like monitoring application, the total number of the sensor nodes that are required can range from 50 to 1000 per region. For instance a home may be containing around two dozen or more of the appliances that may be containing the sensor nodes, and this number is going to rise, if sensor nodes are supposed to be in the furniture and may be in other miscellaneous items. The node density depends on the type of application for which the sensor nodes are being deployed. 


\section{Production costs}

Now, if the cost of the network is more expensive than of the deploying like old time sensors, then the wireless sensor network is not to be said as cost justice. As the sensor networks is comprised of very high density of the sensor nodes, so it is very important to keep in mind the cost for a single node, so as to make equilibrium for the overall cost of the network. It should be kept in mind, that the cost of each sensor node should be as low as possible. But, it is really challenging issue to reduce the cost for the sensor node with keeping its functionalities and the application. Sometimes the sensor nodes has some other units like processors or the GPS or some other costly unit which is necessary, for some application, so in that case, it should be done in a way that the nodes be deployed, may be in a heterogeneous manner or in a way that the production cost is minimum.

\section{Hardware constraints}

As we know that the sensor node comprises of mainly four basic components that are a sensing unit-processing unit, transceiver unit and most important over which all are dependent is the power unit. The Sensing unit is divided into two subparts: sensors and the ADC-analog to digital converter. Some additional hardware can be also needed, which can be in the form of power generator or any special type of sensing unit. The most important of the component in a sensor node is the power unit. Sometime, power unit is attached with the charging unit that can be solar power or may be chargeable. There can be more units, which is totally dependent on applications. In some of the cases sensor are mobile, so there is the need of extra battery power and how the sensors are going to move. Now the issue is to include all the units into a small board so the constraint is to make the units small and to plant it on the board. These nodes should have the ability to consume minimum power, ability to work and operate in high densities, It should have the low production cost, should have the ability to operate unattended and should be autonomous and at last must be adaptive in nature.

\section{E. Sensor network topology}

For the deployment of high number of nodes in a dense behavior it is necessary for the careful handling and maintenance of the topology. Many sensor nodes are deployed in the field. Some of them are inaccessible and unattended sensor nodes that are much prone to the failure. So, there is a need to make the topology change sometime for the maintenance of the overall network. The topology change can be noticed in the three phases.

Pre-deployment phase: The nodes are deployed in unwary manner way sometimes, it is dropped from the airplane or sometimes thrown in inaccessible way or is deployed randomly. There are several means and techniques through which one can easily deploy the sensors, which should be such that it should reduce the installation cost and should have fault tolerance, the capability of self-organization and should eliminate the need of any pre planning.

Post-deployment phase: The Sensor nodes are sometimes statically deployed. As we know that after the deployment the sensor network changes as the topology changes, in terms of position or the reach ability or the availability of power, as per the application and sometimes in malfunctioning and task details. Moreover, the node failure is often a common event and a regular too, due to dead nodes or the less battery power. There are some wireless sensors networks that have highly mobile nodes so the numbers of nodes destroying are supposed to be replaced or because of its mobility, it has to be recharged. So after deployment there is huge possibility for the nodes to die and the need for the post deployment.

Re-deployment of additional nodes phase: In addition after the re organizing of the network and the post deployment there are some times, where there is a need to add, some nodes for the start of a reliable working of the wireless sensor network. Sometimes the protocols defined for the application need the reliability, so there is need for the re-deployment of the sensor nodes to keep on the working of the system.

\section{F. Environment}

In some of the application the Sensor nodes are deployed in a very dense manner, which can be either very close or deployed inside the application. They can be deployed in a unmanned area, like in rural or in ocean, near the volcano, some biological usage, in military services, on battle field, or the large buildings, bridges or any other infrastructure. So we can say that they are mostly in a very remote area because of which it is very important parameter to be known before moving towards any application in wireless sensor networks.

\section{LIFETIME OF WIRELESS SENSOR NETWORK}

The definition of the lifetime of wireless sensor network can be defined in number of ways as discussed below.

a) Number of Alive nodes at certain time describes the Network Lifetime: It depends on the ratio of number of dead nodes. Basically it depends on the application that what is the demand of reliability. As soon as the first node is dead or the number of nodes dead crosses the threshold then the network lifetime is said to be over.

\section{b) Network Lifetime Based on Sensor Coverage [4]:} Sensors are responsible for the coverage and connectivity. Network also depends on the coverage to check if the area which is of interest to the application, is covered or not properly. As soon as the area of interest is no more covered then we say that the network lifetime is decreasing. It is based on the two approaches that are, first is that defined part of the region is at least covered by one sensor or the next definition says that the each 
point in the wireless sensor network is or should be covered by certain defined number of sensors in order to say that the network lifetime is maintained.

c) Network Lifetime Based on Connectivity: [4] the connectivity in the wireless sensor network is another important perspective to define the network lifetime. Connectivity is mainly used in the adhoc networks, in comparison to the sensor networks, as there is no specification of coverage in the adhoc network. Some of the definitions that are given by researchers like Blough and Santi defines that the network lifetime is maintained till some of the defined percentage or the largest connected network is below some threshold. Some define the network lifetime in the number of the packet that is being sent to the sink with considering the reliability.

d) Network Lifetime Based on Sensor Coverage and Connectivity; In this case the network lifetime is defined in terms of both the network connectivity and the coverage. It is checked that if the coverage reaches below certain threshold and the number of packets that are being sent to the sink for the connectivity, unless and until both of the parameters are not fulfilled, then only we say that the network lifetime is maintained. e) Network Lifetime Based on Application Quality of Service Requirements: The sensor network is more application specific. The network is said to be live, until the requirements of the application is being satisfied by the network. The second consideration is that it should not give unreliable result on event detection ratio, so the quality of service is maintained till the network lifetime is maintained in terms of the reliability for the application.

\section{Heterogeneous WIRELESS SENSOR NeTWORK}

The major impact of the Heterogeneous network in wireless sensor network is reflected through the Fig.3.

Major highlights of heterogeneous over homogeneous is Better network lifetime: Heterogeneity can be in terms of the link or battery or computation [5]. If we apply any of the heterogeneity then we can enhance the network lifetime. Reliability in Data Transmission: The reliability will be maintained as the heterogeneity will not let the node to die very soon and the computation heterogeneity will help to make the report fast. Decrease in data transmission Latency: The channel heterogeneity can help to decrease the time at which the report is send or any event detection report.

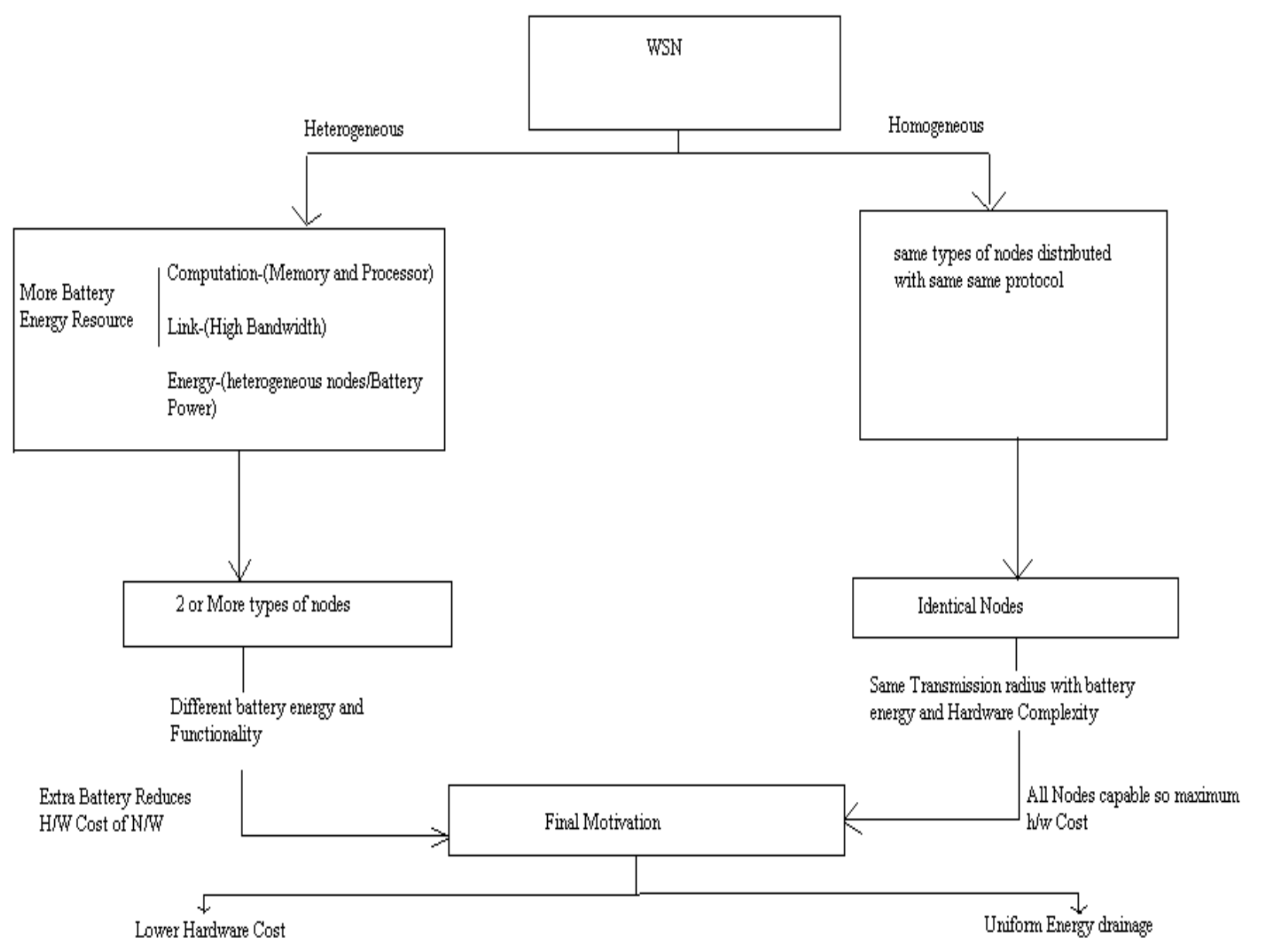

Fig. 3: Comparison of Homogeneous to Heterogeneous WSN 


\section{PERFORMANCE MEASUREMENT}

The performance of the whole system can be calculated by-

a) Network Lifetime- Lifetime will tell the time till which the data was correct in terms of reliability and the generated event report.

b) Cluster Head per round- The number of cluster head that are chosen. The number of times and according to the area number of clusters that are chosen helps to provide the performance of the network [6].

c) Number of Alive nodes- The time, till which the number of nodes are alive and does not cross the threshold or they are up to coverage and connectivity, through these factors the performance of the system can be calculated.

d) Throughput- The number of Packets that are being transferred or delivered or reported to the sink or the event report gives the performance of the networks.

\section{ENERGY ORIENTED STRATEGIES}

Energy oriented Strategies [7] are-:

\section{A. Cluster based approach}

In the wireless sensor network, the sensor nodes form a cluster head. The nodes in the cluster are termed as cluster member who sends the data to the cluster head and then this cluster head forwards the respective data to the sink or the base station as shown in Fig. 4. By the cluster head formation there is scalability in the network and also it provides reliability up to some extent in the network. There are different techniques, to choose the cluster head which is also a major problem. For example one of the schemes for clustering is by using connected graph [8]. Here the clustering is innovated and formed in three steps, which are cluster head election, clusters formation and clusters routing. The connected graph is formed for efficient data flow. The main attributes that affects the selection or the behavior of cluster head are the Mobility of nodes, the role of the node and the cluster head and the type of node that has to be cluster head.

\section{B. $\quad$ Chain based approach [7]}

In the chain based approach the node tends to receive and transmit the data to its neighbors. The data follows the chain type of structure which in turn leads to the Base station. In this way the energy of the network is distributed. In this case the sensor nodes are randomly deployed. The chain can be formed by two means, one is that the node itself can form the chain by applying different algorithms like Greedy algorithm or in other case the base station will decide the chain to be followed.

In any case if any node dies, then the chain is remade. With respect to the aggregation of the data, what happens is that the node collects its data and forwards it to the next node in the chain, which aggregates the data of it and then forwards this combined data.

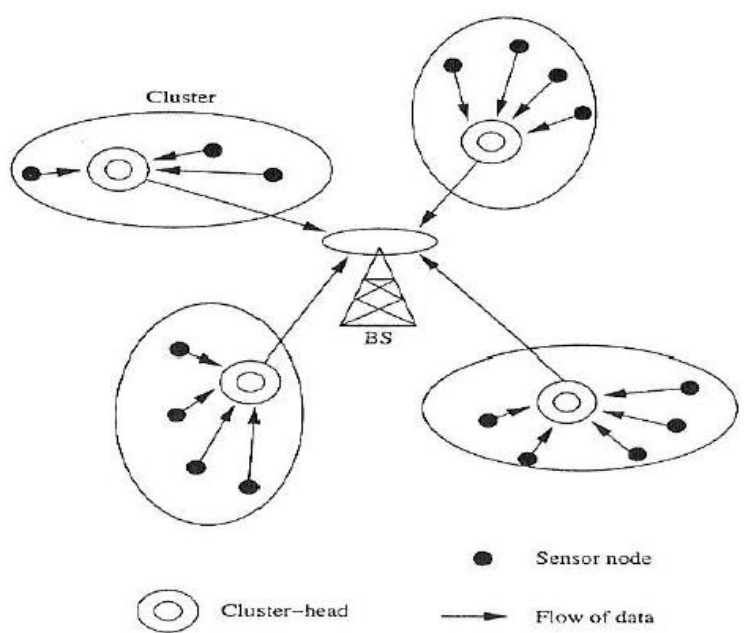

Fig. 4: Clustering in WSN

\section{Self-Organizing Protocol (SOP) [7]}

In the self-organizing protocol the sensor nodes are mobile. Some of the sensor nodes become the router and some out of them monitors the environment. The node which is router is usually stationary. The mobile node follows different sort of algorithm to support different parameters like fault tolerance. The algorithm can be like spanning tree graph for the mobility. These types of protocols are good where the application is moreover application specific.

The advantages of the clustering approach are as follows: [9] (1) the size of the routing table can be reduced, (2) The bandwidth is saved as the data is only send to the cluster head, (3) The cluster head has to apply different strategies to increase the overall lifetime of the sensor network, (4) There will be no collision of packets as only the cluster head will send the data, (5) Cluster head can adopt scheduling strategies. The properties and characteristics that show the properties inside the cluster or to the inter clusters can be defined like Cluster Count: In some of the cases, according to the requirement, the cluster heads are decided, at an earlier stage only. The algorithms generally work in a way such that the choice of cluster head is random or according to the weight or heterogeneity.

\section{Intra cluster Topology}

In some cases the sensor has the duty to report to Cluster head. But in few cases the cluster head follows multi hop. By this the sensors use multi hop communication to report to cluster head. Connectivity of $\mathrm{CH}$ to BS: There can be direct link or it can happen that the cluster head chooses the multi hop communication. Routing is discussed as a part of the link layer where multi hop is not fully considered. [10] Several studies mention, that multi hop for WSN helps for achieving network performance, reducing energy consumption and 
extending network lifetime. The cluster head has to report to sink. It uses any of these ways to communicate to sink. Different clustering schemes have different type of techniques [11]. We can differentiate them on the basis of Mobility, Node Types and Role. The CH Selection Based on [12] Initial Energy, Residual Energy, Energy Consumption Rate and Average Energy of the Network. Some of the Energy Efficient Clustering Protocol for HWSN are-

Energy Efficient Heterogeneous Clustered Scheme: [13] it uses the approach of hierarchal WSN. It uses weight for calculating the probabilities and the cluster head respectively and accordingly selects the cluster head. Distributed Energy Efficient Clustering Algorithm for HWSN: [14] It chooses the Cluster head on the basis of energy of node and by calculating this energy over the total energy of the system.

Distributed Energy Balance Clustering Protocol for $H W S N$ : [15] It has multilevel heterogeneity like two level heterogeneity. It selects the cluster head on the basis of same as by calculating the energy of the node and the total of the system.

Stability oriented Clustering Protocols for HWSNs [4]: It is the second phase for the cluster head formation. It, is the time for any application, before the first node dies. Base Station Initiated Dynamic Routing Protocol: [12] It uses heterogeneity in terms of the nodes, in this the node which has the better efficiency is selected as the cluster head.

Routing Protocol for Balancing Energy Consumption in HWSN: [12] It is based on the residual energy for the selection of the cluster head. Moving ahead of this, the next protocol came that was zone based, in which the cluster head was selected as per the closeness from the base station.

Distributed election clustering protocol (DECP) for HWSN: [12] It is based on the minimal cost. The nodes broadcast their energy and then the other node receives the energy and compares with it and then again broadcast this way the cluster head is selected. This message is floated then and all the nearest nodes are asked to get connected to the cluster head.

Steady clustering scheme for HWSN: It follows the initial energy of the nodes. It has two steps that are data transmission stage and the grouping stage. Base station, broadcasts a message i.e group head message then, after receiving this message, every node sends back the receiving message with its own id and also its initial energy. Now the base station selects the group head and again broadcast it for the nodes to listen to it and get joined to that node.

\section{CONCLUSION}

Due to some of the stringent constraints in wireless sensor network like lifetime, computation etc. There are many applications, which still need to be evolved. Many researchers' are working in this domain to evolve a technique which can increase the overall lifetime of the system. As per the survey if heterogeneous nodes are taken on account and the appropriate scheme is taken for the sleep- awake and data transfer, then it will definitely have a huge impact on overall lifetime of WSN as compared to homogeneous system. Through the study, it can be deduced that the optimal way of heterogeneity with respect to extending lifetime of the network, is to have node battery heterogeneity, rather than of link or computational heterogeneity.

\section{ACKNOWLEDGMENT}

We would like to give sincere thanks to the Wireless sensor lab of IIIT, Allahabad- India and NIT, Hamirpur for carrying out the respective study for this paper work .Prof. Saddek Bensalem and Prof. Joseph Sifakis from Verimag Lab, Grenoble- France for providing us with the important concepts in Wireless Sensor Network domain to come up with facts inculcated in this paper.

\section{REFERENCES}

[1] S.Barani, C.Gomathy, "Power Aware Heterogeneous WSN", 2011 International Conference on Network and Electronics Engineering, 2011.

[2] Falko Dressler and Isabel Dietrich, "Lifetime analysis in heterogeneous sensor network", In Proceedings of the 9th EUROMICRO Conference on Digital System Design (DSD'06) IEEE On page(s): 606 - 616.

[3] I.F. Akyildiz, W. Su, Y. Sankarasubramaniam, E. Cayirci, "Wireless sensor networks: a survey", in Elsevier Science B.V, 2002.

[4] Isabel Dietrich and Falko Dressler, "On the Lifetime of Wireless Sensor Networks", ACM Transactions on Sensor Networks, Vol. 5, No. 1, Article 5,: February 2009.

[5] Akyildiz, W. Su, Y. Sankarasubramaniam, and E. Cayirci, "A survey on sensor networks". IEEE Communications Magazine, 40(8):102-114, August 2002.

[6] Enrique J. Duarte- Melo, Mingyan Liu, "Energy-Efficient Cluster Formation in Heterogeneous Wireless Sensor Networks: A Comparative Study", in Advanced Communication Technology 2011 IEEE page 21-25, 2011.

[7] Shilpa Mahajan, Dr. Jyoteesh Malhotra, "Energy Efficient Control Strategies in Heterogeneous Wireless Sensor Networks: A Survey", in International Journal of Computer Applications (0975 - 8887) Volume 14- No.6, page(s) 3137, February 2011.

[8] Ye M., Li C., Chen G., and Wu J., "EECS: An Energy Efficient Clustering Scheme in Wireless Sensor Networks", in Proceedings of 24th IEEE International Performance, Computing, and Communications Conference (IPCCC'2005), pp. 535-540, 2005.

[9] Shariar Imtiaz, Md. Mosaddek Khan, Md. Mamun-orRashid, Md. Mustafizur Rahman, "Improved Adaptive Routing for Multihop IEEE 802.15.6 Wireless Body Area Networks", International Journal of Intelligent Systems and Applications (IJISA), ISSN: 2074-904X, ISSN: 20749058. 
[10] Kumar D., Trilok C., Patel R., "A Two Tier Data Aggregation and Clustering Scheme for Heterogeneous Sensor Networks", in Proceedings of the IEEE International on Advance Computing Conference, Patiala, pp. 2053-2058, 2009.

[11] Vivek Katiyar, Narottam Chand, Surender Soni, "Clustering Algorithms for Heterogeneous Wireless Sensor Network: A Survey", in International journal of applied engineering research, Dindigul, Volume 1, No 2, 2010.

[12] Dilip Kumar, Trilok C. Aseri, R.B. Patel," EEHC: Energy efficient heterogeneous clustered scheme for wireless sensor networks", Computer Communications 32 (2009) 662-667, 20 November 2008.

[13] Chong, C., Kumar, S., "Sensor Networks: Evolution, Opportunities, and Challenges", Proc. of the IEEE, Vol. 91, No. 8, 2003.

[14] Feng Li, Liuhong Huang, "CRPCG-Clustering Routing Protocol based on Connected Graph", International Journal of Intelligent Systems and Applications (IJISA) ISSN: 2074-904X, ISSN: 2074-9058.

[15] R.S. Marin-Perianu, J. Scholten, P.J.M. Havinga and P.H. Hartel, "Cluster-based service discovery for heterogeneous wireless sensor networks", International Journal of Parallel, Emergent and Distributed Systems, 2005.

[16] Enrique J. Duarte-Melo, Mingyan Liu, "Analysis of Energy Consumption and Lifetime of Heterogeneous Wireless Sensor Networks" in Global Telecommunication conference, 2002. IEEE .On page(s): 21 - 25 vol.1, 2002.

[17] I. Akyildiz, W. Su, Y. Sankarasubramaniam, and E. Cayirci, "A survey on sensor networks". IEEE Communications Magazine, 40(8):102-114, August 2002.

[18] Marin Perianu, R.S. Scholten, J Havinga, P.J.M. and Hartel P.H."Cluster based service discovery for heterogeneous wireless sensor networks", International Journal of Parallel,
Emergent and Distributed Systems, 23 (4): pp 325346, (2008).

\section{Authors' Profiles}

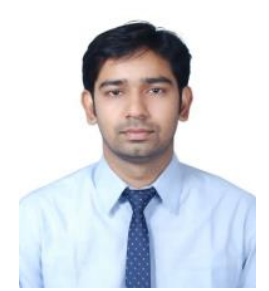

Ritesh Kumar Singh was born in Uttar Pradesh, India, in 1988. He received his B.E and ME degree in Computer Science and Engineering from HCST, Mathura and IIIT, Allahabad India in 2010 and 2012 respectively. In 2012, he joined Verimag Laboratory in Grenoble, France to do a project under Prof. Joseph Sifakis. After successful completion of the project he joined LG research and development lab in Bangalore, India. His research interests include routing techniques, Wireless sensor Networks, wireless communication and Model checking.

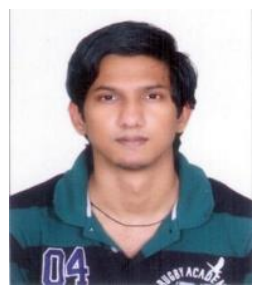

Ankit Bhadoria was born in Madhya Pradesh, India, in 1990. He received his Bachelor's degree in Computer Science and Engineering from National Institute of Technology Hamirpur (India) in the year 2012. In 2011, he joined IIIT Gwalior for a period of 3 months, to do a project under Prof. Anupam Shukla. From 2012, he is employed by LG research and development lab in Bangalore, India (LGSI). His Research interests include Artificial Intelligence, Robotics and Algorithms.

How to cite this paper: Ritesh Kumar Singh, Ankit Bhadoria,"Lifetime of Sensor Network by Exploiting Heterogeneity- A Survey", IJMECS, vol.6, no.7, pp.47-54, 2014.DOI: 10.5815/ijmecs.2014.07.07 